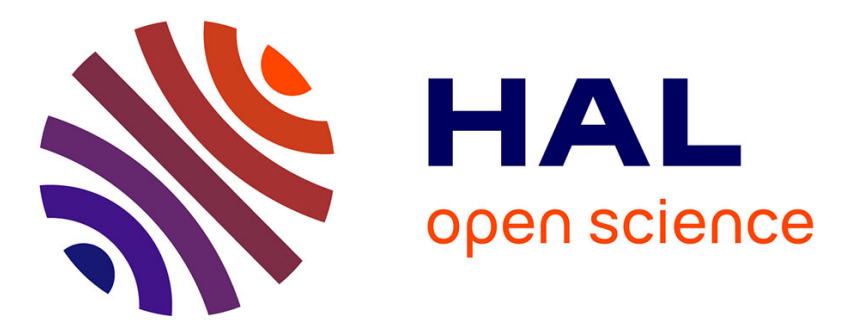

\title{
Construction de la plaine rizicole du Népal: sous le prisme de la gestion de l'eau et des processus de territorialisation dans le Sunsari
}

\author{
Marie-amélie Candau, Romain Valadaud, Olivia Aubriot
}

\section{To cite this version:}

Marie-amélie Candau, Romain Valadaud, Olivia Aubriot. Construction de la plaine rizicole du Népal: sous le prisme de la gestion de l'eau et des processus de territorialisation dans le Sunsari. Espace Géographique, 2015, 44 (2), pp.160-173. 10.3917/eg.442.0160 . halshs-01673749

\author{
HAL Id: halshs-01673749 \\ https://shs.hal.science/halshs-01673749
}

Submitted on 27 Nov 2020

HAL is a multi-disciplinary open access archive for the deposit and dissemination of scientific research documents, whether they are published or not. The documents may come from teaching and research institutions in France or abroad, or from public or private research centers.
L'archive ouverte pluridisciplinaire HAL, est destinée au dépôt et à la diffusion de documents scientifiques de niveau recherche, publiés ou non, émanant des établissements d'enseignement et de recherche français ou étrangers, des laboratoires publics ou privés. 


\title{
Construction de la plaine rizicole du Népal : sous le prisme de la gestion de l'eau et des processus de territorialisation dans le Sunsari
}

\author{
Auteurs : CAndau Marie-Amélie, VAladAud Romain et Aubriot Olivia ${ }^{1}$ \\ Doctorante Paris Ouest Nanterre et CNRS (CEH), Etudiant M2 de Trinity College of Dublin, \\ Chargée de recherche CNRS (CEH) \\ Centre d'Etudes Himalayennes, 7 rue Guy Môquet, 94800 Villejuif \\ marieameliecandau@gmail.com; rvaladaud@hotmail.fr; olivia.aubriot@cnrs.fr
}

\begin{abstract}
Résumé
La plaine du Népal, après avoir été marginalisée, a connu des transformations spectaculaires lors des cinquante dernières années, pour devenir le grenier à grain du pays. Or cette construction se révèle être le fruit d'une idéologie ancienne, qui lança l'instauration de politiques foncières, puis d'aménagements étatiques. L'article étudie les modifications induites dans le rapport à l'eau et à la terre, et dans la territorialité des différents groupes d'acteurs. Il analyse la co-construction de l'organisation hydraulique par les dynamiques sociales, sa transcription territoriale et montre que la situation de marge explique certains conflits sociaux visibles dans la gestion de l'eau.
\end{abstract}

Mots-clés : conflits sociaux, eau, foncier, marges, territorialité

\section{Introduction}

Les projets de territorialité institutionnelle, qu'il s'agisse de politiques foncières, d'aménagement ou de développement d'infrastructures, induisent des mutations territoriales. Certains les décrivent comme une complexité caractérisée par un «déploiement simultané de différentes territorialités » et un « rapport à l'espace correspondant à différentes logiques et pratiques » (Moine, 2006 ; Giraut, 2013). D'autres auteurs analysent ces mutations en tant que fragmentations et recompositions territoriales, comme en Himalaya indien où une «ethnicisation des territoires », dénommée aussi "processus d'ethno-territorialité », s'y observe dans les marges créées dès la période coloniale britannique (Smadja, 2013). Bien que n'ayant jamais été colonisé, le Népal présente également cette tendance. Il traverse en effet une période de revendications ethniques et identitaires par les populations historiquement marginalisées, notamment celles de la plaine.

Le contrôle de cet espace par l'Etat centralisé est indispensable au vu des enjeux démographiques, économiques et géopolitiques que représente la plaine : passée en un demisiècle d'un front pionnier à un « espace plein », elle accueille aujourd'hui plus de la moitié de la population nationale (sur 17\% du territoire) et est le grenier à grain (riz, blé) du pays. Ces transformations spectaculaires sont le fruit d'investissements et aménagements étatiques lourds, de l'accueil continu de migrants des montagnes, et d'une volonté d'annexer pleinement cet espace productif. Le district de Sunsari, dans l'est de la plaine, et sur lequel s'appuie notre étude, illustre parfaitement ces changements du fait de l'implantation de grandes structures hydrauliques étatiques (barrage, digues, vaste système d'irrigation) et d'un flux incessant de populations des montagnes.

Pour aborder ces mutations, nous envisageons la territorialité de manière dynamique (Raffestin, 1986), en donnant une place privilégiée aux acteurs (Bonerandi, 2001). En effet, les

\footnotetext{
${ }^{1}$ Les auteurs tiennent à remercier l'Agence Nationale de la Recherche pour son soutien financier au projet 'TerreEau' dans lequel le présent travail s'inscrit (ANR-12-AGRO-0002-02).
} 
infrastructures hydrauliques sont souvent étudiées avec une approche technique (d'efficience), économique (de rentabilité) ou institutionnelle (de la gestion participative). Dans cet article, nous adoptons une vision constructiviste : la réalité sociale d'une situation donnée est le produit d'interactions sociales entre acteurs intégrant les contraintes de leur milieu. Comme Jeanne Riaux (2015) l'expose, l'analyse des différents discours des acteurs d'un système hydraulique est indispensable pour saisir les prétendus dysfonctionnements hydrauliques. Dans notre cas, cette analyse se centre autour de la co-construction entre nature, société et technique, les infrastructures hydrauliques étant, malgré leur aspect fixe, remodelées par les dynamiques sociales (Marié, 1987 ; Trottier et Fernandez, 2010). Pour étudier celles-ci, les auteurs ont effectué plusieurs séjours de terrain dans le Sunsari ${ }^{2}$ et réalisé, auprès d'acteurs divers, des enquêtes principalement qualitatives, enregistrées et retranscrites.

Notre hypothèse est alors que tout projet de territorialité institutionnelle induit des mutations territoriales qui, dans le cas d'un aménagement hydraulique se répercutent dans la gestion de l'eau. Partant de cette hypothèse, nous montrons que l'étude des systèmes hydrauliques du district de Sunsari permet d'accéder aux processus de reterritorialisation à l'œuvre dans ce district.

Pour cela, nous abordons en première partie la manière dont les politiques foncières de l'Etat népalais ont contribué à faire de la plaine un pilier économique du pays, tout en laissant cet espace à la marge socialement et politiquement. Nous verrons que l'Etat s'est peu impliqué dans la gestion locale de l'eau au sein du district étudié. Un basculement s'opère en 1950, lors de l'ouverture aux capitaux internationaux du pays. Notre deuxième partie décrit la transformation du territoire du Sunsari à travers l'implantation d'infrastructures hydrauliques tout en étudiant leurs logiques productives et stratégiques. Dans une troisième partie, nous montrons que la co-construction de l'évolution du système hydraulique est guidée par les dynamiques sociales et les discours qui les accompagnent. Ainsi, nous verrons comment la gestion de l'eau permet d'exprimer des oppositions sociales et politiques, et d'exposer une territorialité multiple au sein d'un même espace.

\footnotetext{
${ }^{2}$ Selon les auteurs : 18 mois, 4 mois ou plusieurs petits séjours d'un mois.
} 


\section{Projet de territorialité institutionnelle à travers les politiques foncières, dès l'unification}

Située au pied de l'Himalaya, dans le prolongement de la plaine gangétique, la plaine du Téraï (Carte 1) est souvent présentée comme un espace qui, au Népal, était peu exploité, impaludé et couvert de forêts jusqu'en 1951. Ce discours d'un espace forestier est toutefois à nuancer et à analyser par rapport au projet politique de contrôle du territoire et de ses habitants.

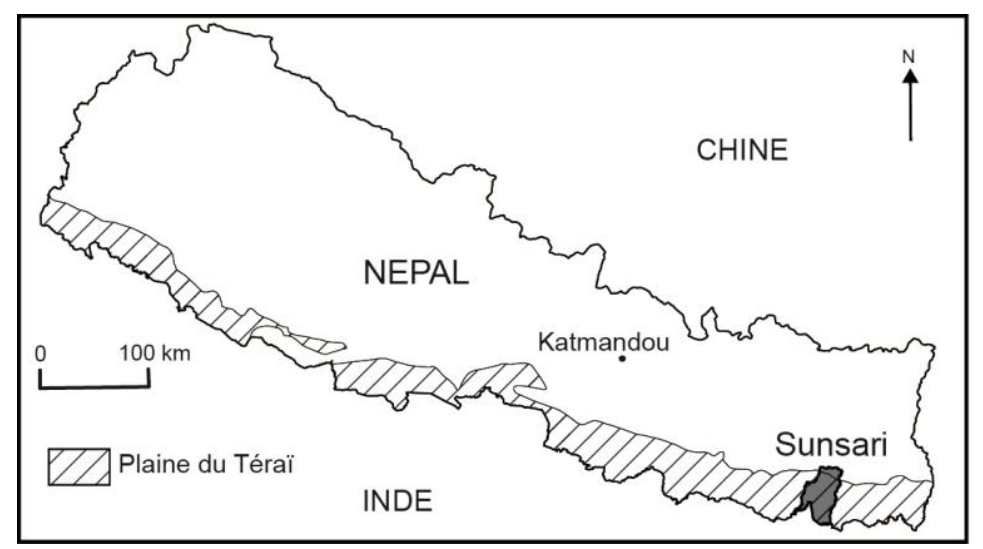

Carte 1 : Localisation de la plaine et du district de Sunsari au Népal Réalisation: J. Picard (CNRS-CEH)

\subsection{La plaine : historiquement un front pionnier de marge}

Le roi de Gorkha, de haute caste hindoue, a unifié le Népal en 1769 en conquérant les différents royaumes alors en place. Il est à l'origine de la construction de l'État népalais, centralisé, dont la capitale est installée à Katmandou, dans les montagnes. La plaine fut alors traitée comme une colonie interne par le pouvoir central (Gaige, 1975) et donc mise en situation de marge soit, selon la définition de Frédéric Giraut (2005, p. 174) "en périphérie d'un espace administratif englobant, où le sentiment d'appartenance est atténué, une marge se situant notamment par rapport à un centre". Couverte de forêts et de zones humides, impaludée de juin à octobre durant la mousson, elle servit de barrière naturelle contre les Britanniques. Toutefois, l'État des XVIII ${ }^{\mathrm{e}}$ et XIX ${ }^{\mathrm{e}}$ siècles, qui avait pour idéologie un Népal peuplé et rizicole (Ramirez, 2003), nota très vite l'intérêt de son exploitation économique, et incita dès la fin du XVIII siècle à sa colonisation par des populations d'Inde du Nord ${ }^{3}$ (Regmi, 1978b ; Dahal, 1983). Le Téraï était déjà pourtant habité par des Tharu, ethnie de la plaine, riziculteurs et pêcheurs, réputés bons défricheurs en Inde du temps des Britanniques (Krauskopff, 2000), ainsi que des populations de castes de la plaine dont il est difficile de connaître la date d'implantation (Gaige, 1975, p. 61). Ces dernières sont similaires aux populations indiennes qui ont été incitées à s'installer, et toutes sont aujourd'hui dénommées Madhesi.

De 1814 à 1860, la frontière entre l'Inde et le Népal a changé de position à plusieurs reprises, imposant aux populations de dépendre alternativement de l'un ou l'autre pays et donc de subir une ligne de discontinuité mouvante ${ }^{4}$. Nous retiendrons pour notre propos que lors de la guerre contre les Britanniques en 1814 est né, parmi les Gorkhali, un ressentiment contre les Madhesi du fait de leur alliance avec les Britanniques. En outre, peu de temps après leur arrivée au pouvoir les Premiers Ministres Ranas (1846-1950) édictent le premier code civil népalais,

\footnotetext{
${ }^{3}$ Il ne pouvait demander aux populations des montagnes qui considéraient la plaine comme la « vallée de la mort » (Dahal, 2013)

${ }^{4}$ Cette caractéristique de coupure créant une discontinuité permet aussi d'appréhender la plaine comme un confins, selon la définition de Frédéric Giraut (2005).
} 
puissant outil de contrôle socio-territorial (Adhikari, 1984) qui institutionnalise la hiérarchie des populations : il place en position dominante les hautes castes (et parmi elles, celles des montagnes sur celles de la plaine) et ignore de nombreux groupes Madhesi (Bennett et al., 2008, p.3), les marginalisant ainsi totalement.

En 1951, le Téraï n'est pas le « confins vide », inhabité qui est souvent décrit, puisque 35\% de la population du pays (soit 2.9 millions de personnes) y réside déjà et la cultive (Gurung, 1998), essentiellement des populations (ethnies et castes) de la plaine.

\subsection{Les transformations territoriales et sociales engendrées par les politiques foncières}

Avec l'unification, le roi devient le propriétaire de la terre qu'il alloue contre services rendus à une élite naissante (administrative, militaire) dont il s'assure ainsi la loyauté. Parallèlement le pouvoir central a besoin de relais locaux pour la collecte des taxes soit, dans la plaine, des Tharu et hautes castes Madhesi qui obtiennent un statut social local plus élevé. Ce modèle de type féodal-centralisé (Sugden, Gurung, 2012) a ainsi induit des transformations majeures dans le système social. À l'instar de Gisèle Krauskopff (2000, p. 38), on peut se demander si la volonté de l'État népalais n'était pas, en faisant appel à des Indiens, de briser la cohésion sociale des Tharu. Le modèle foncier a également figé le territoire et ses habitants : en deux siècles, les Tharu passent de semi-nomades à paysans sédentaires sur des terres devenues propriété du roi hindou ou de ses délégués (Krauskopff, 1989, p. 15).

Ce système de tenures et de taxes foncières assure le revenu de l'État (Regmi, 1978a) et notamment celui nécessaire au soutien des guerres expansionnistes ${ }^{5}$ des Gorkhali de la fin du $\mathrm{XVIII}^{\mathrm{e}}$ : les paysans de la plaine «approvisionnaient le reste du pays en riz et autres denrées de valeur» (Meyer, 2000, p. 16). Le Téraï constitue un pilier dans l'établissement du pouvoir central. Les Ranas affermissent les politiques et à partir de 1860, usant de diplomatie avec les Britanniques et n'ayant donc plus besoin de la plaine comme barrière, ils encouragent à nouveau sa mise en valeur (Gaige, 1975, p. 60). C'est d'ailleurs à cette époque que dans le Sunsari, d'après nos entretiens, la première vague de défrichement (par des Tharu, dans la partie nord de la plaine et des Madhesi dans le sud) aurait commencé. Une deuxième vague aurait eu lieu dans les années 1920-1930 dans le centre du district, Madhesi et Tharu s'installant simultanément. Tous provenaient du district voisin de Saptari, plus anciennement peuplé et situé à l'ouest, ou de l'Inde, au sud. Bien que les migrations nous aient souvent été présentées comme spontanées par les populations, on peut émettre l'hypothèse que la majorité des implantations dans le Sunsari sont liées au renforcement des politiques de mise en valeur de la plaine, c'est-à-dire aux ordres de défrichement dont certains nous ont fait mention.

Tharus et Madhesi ont construit des systèmes d'irrigation de petite taille, déviant l'eau des cours d'eau locaux et réparant collectivement prises d'eau sommaires et canaux en terre. Ils s'organisaient en outre entre hameaux dépendant d'un même cours d'eau pour gérer la distribution de la ressource. D'après nos enquêtes, cette eau mettait ainsi en lien des Tharu pour la partie centrale et nord du district, et des Madhesi pour la partie méridionale. Elle a participé à la construction du territoire par les nouveaux arrivants qui se sont appropriés les ressources de cet espace récemment défriché. Aucun indice ne laisse penser à une ingérence de l'État dans la gestion locale de l'eau d'irrigation, ce qui concorde avec les analyses sur l'absence d'implication directe de l'État qui se contenta d'inciter à l'irrigation et de stabiliser les droits de propriété (Pradhan, 1990 ; Aubriot, 2004).

Au milieu du $20^{\mathrm{e}}$ siècle, le Népal fait le choix de rendre la plaine attractive afin d'y installer des populations des montagnes et des migrants népalais refoulés d'Assam et de Birmanie (Dahal 2013, p.158) ainsi que d'ouvrir le pays aux personnes et investissements étrangers. De

\footnotetext{
${ }^{5}$ Vers le Tibet, le Sikkim et le Kumaon
} 
nombreux programmes sont alors mis en place : éradication du paludisme, projets d'installation des migrants, aménagements d'infrastructures routières et hydrauliques, implantation d'hôpitaux et d'écoles, et réforme agraire (en 1964) qui abolit les statuts fonciers privilégiés. Ce sont des transformations massives pour la plaine auxquelles s'ajoutent les migrations depuis les montagnes - en 2001 de tels migrants représentent $37 \%$ de la population en plaine (Dahal, 2013, p. 159). C'est dans ce contexte que prend place la transformation du Sunsari, principalement autour d'aménagements sur la rivière Koshi, qui le borde à l'ouest.

\section{Processus de territorialisation étatique par les grands aménagements hydrauliques dans la plaine : le cas de Sunsari}

L'aménagement de la Koshi, rivière impétueuse, difficile à dompter et très chargée en sédiments, fut l'objet en Inde de nombreuses controverses parmi les ingénieurs Britanniques. Après l'Indépendance, les travaux d'endiguements sont initiés dès 1953 (Mishra, 2008). Ce pays, profitant de l'ouverture du Népal aux investissements étrangers, a étendu sa vision aménagiste, transformant drastiquement plusieurs districts de la plaine népalaise suite à la signature d'accords bilatéraux. Le district du Sunsari est marqué par celui concernant la Koshi, signé en 1954. Les années 1950-1960 marquent au Népal l'emprise de l'aide internationale dans la structuration du secteur de l'irrigation: création du Département de l'irrigation (DoI) centralisé et entrée dans la période hégémonique des aménagements lourds.

\subsection{Transformations de la géographie du district}

a. Transformation matérielle du territoire

L'accord de la Koshi permet à l'Inde d'endiguer cette rivière au Népal et d'y construire, près de la frontière, un barrage. L'hydrologie est perturbée (carte 2) : le lit est dorénavant contenu par les digues; le barrage frontalier permet la dérivation de l'eau en Inde dans deux canaux d'irrigation latéraux (le canal de l'ouest délivre toutefois un peu d'eau au Népal, dans le district de Saptari). Se développe dans les années 1970 l'endiguement des autres rivières du Sunsari, les digues étant perçues comme la réponse ultime aux problèmes d'inondation, eux-mêmes nouvellement pris en considération au Népal.

S'y ajoute la construction, en plusieurs étapes, du plus grand système d'irrigation du Népal, le Sunsari-Morang Irrigation System (SMIS) programmé pour couvrir 64000 ha dans les districts de Sunsari et Morang. L'Inde ne bénéficie nullement de cet aménagement mais, du fait de l'accord bilatéral, a réalisé les premières constructions : prise d'eau sur la Koshi et canal de Chatara qui s'écoule d'ouest en est. Achevé en 1975, ce premier tracé fut très vite inopérant pour diverses raisons (déplacement de la Koshi, sable excessif dans le canal, mauvaise qualité de construction). Ce n'est qu'entre 1978 et 2001 que le projet prend réellement forme, à l'aide du financement de la Banque Mondiale : la prise d'eau est déplacée, le canal principal est réhabilité et vingt canaux secondaires sont construits selon l'orientation nord-sud, quadrillant l'espace entre le canal de Chatara et la frontière indienne. Le SMIS vient supplanter les anciens systèmes d'irrigation locaux dans cet espace qui couvre $84 \%$ de la plaine du district ${ }^{6}$. Très vite la plaine a été entièrement cultivée : on n'y trouve plus d'espaces de pâturages, ni de forêt et les rizières sont à perte de vue.

\footnotetext{
${ }^{6}$ Le district de $1200 \mathrm{~km}^{2}$ est composé à $66 \%$ de plaine, essentiellement rizicole, de $7 \%$ du lit de la rivière, et des premiers reliefs qui accueillent la forêt et la ville de Dharan (carte 2).
} 


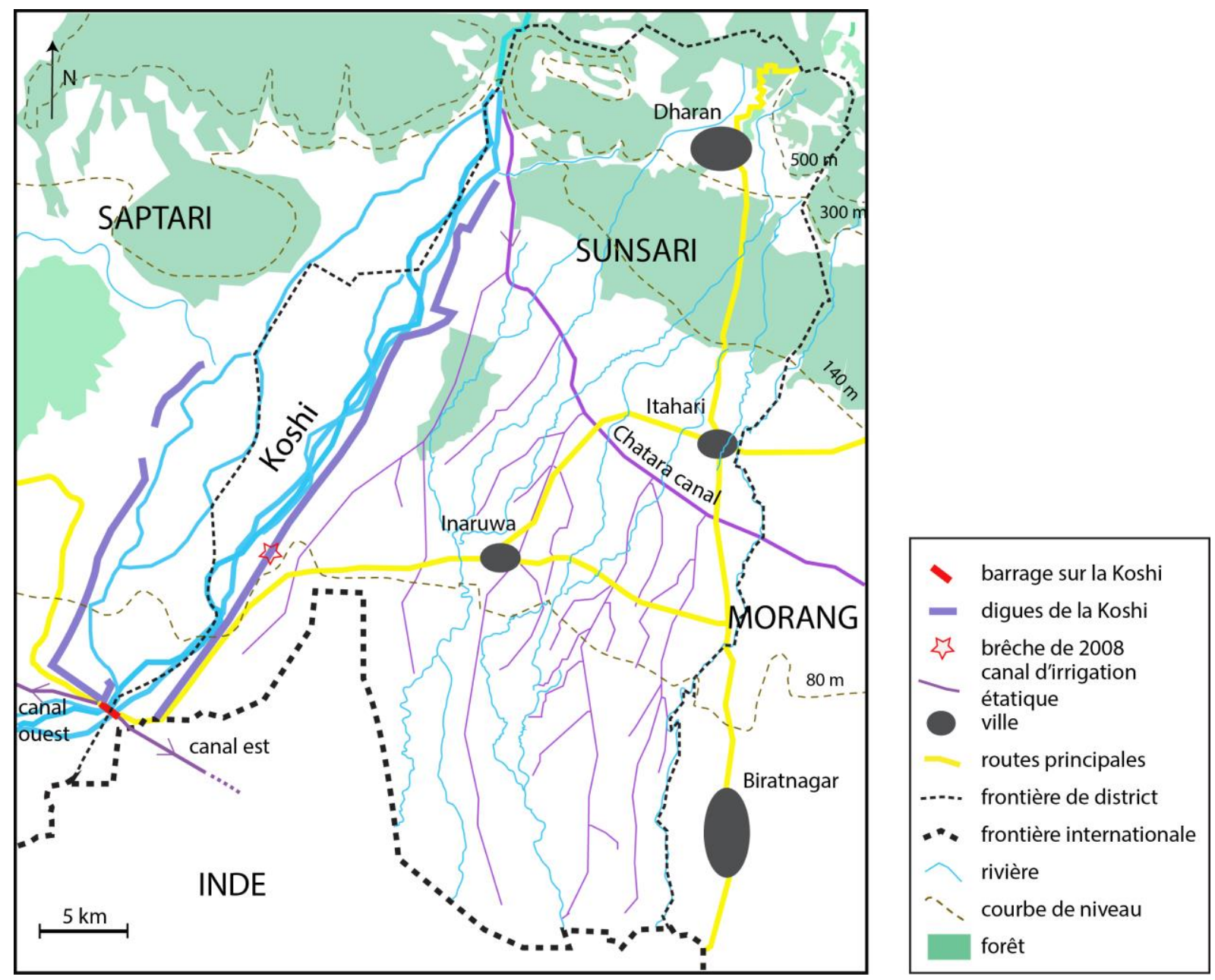

Carte 2 : Principaux aménagements étatiques dans le district de Sunsari

(La limite de la forêt représente approximativement la rupture de pente entre la plaine et la montagne)

Réalisation : O. Aubriot (CNRS-CEH)

\section{b. Transformations démographiques}

La population de cette région ${ }^{7}$ a été multipliée par 7 entre 1951 et 2011 , du fait de la forte croissance démographique naturelle - la population du Népal a triplé pendant cette période mais aussi des nombreuses migrations depuis les montagnes, qui n'ont cessé de se poursuivre depuis les années 1960. En 2011, la densité est de 600 hab./ $/ \mathrm{km}^{2}$ dans le district de Sunsari et les migrants y représentent $44 \%$ de la population, contre $37 \%$ de Madhesi et $18 \%$ de Tharu et autres ethnies indigènes ${ }^{8}$. La carte 3 montre que les Madhesi occupent principalement le sud du district, tandis que les Tharu sont davantage dans l'est et le nord de la plaine et que les populations des montagnes, dénommées Pahari dans la plaine, se sont installées dans la moitié nord du district, peuplant aujourd'hui majoritairement les centres urbains. Dans la campagne, les Pahari se répartissent à la périphérie ou entre les hameaux Tharu; les quelques-uns à bénéficier du SMIS (car installés au sud du canal de Chatara) se retrouvent dans les parties amont des canaux d'irrigation.

\footnotetext{
${ }^{7}$ Le district de Sunsari ayant été créé en 1962, nous avons pris les chiffres de celui dénommé Biratnagar en 1951 qui correspond aux districts actuels de Sunsari et Morang (passage de 0,23 à 1.72 millions de personnes).

8 Nos calculs d'après les données du recensement.
} 


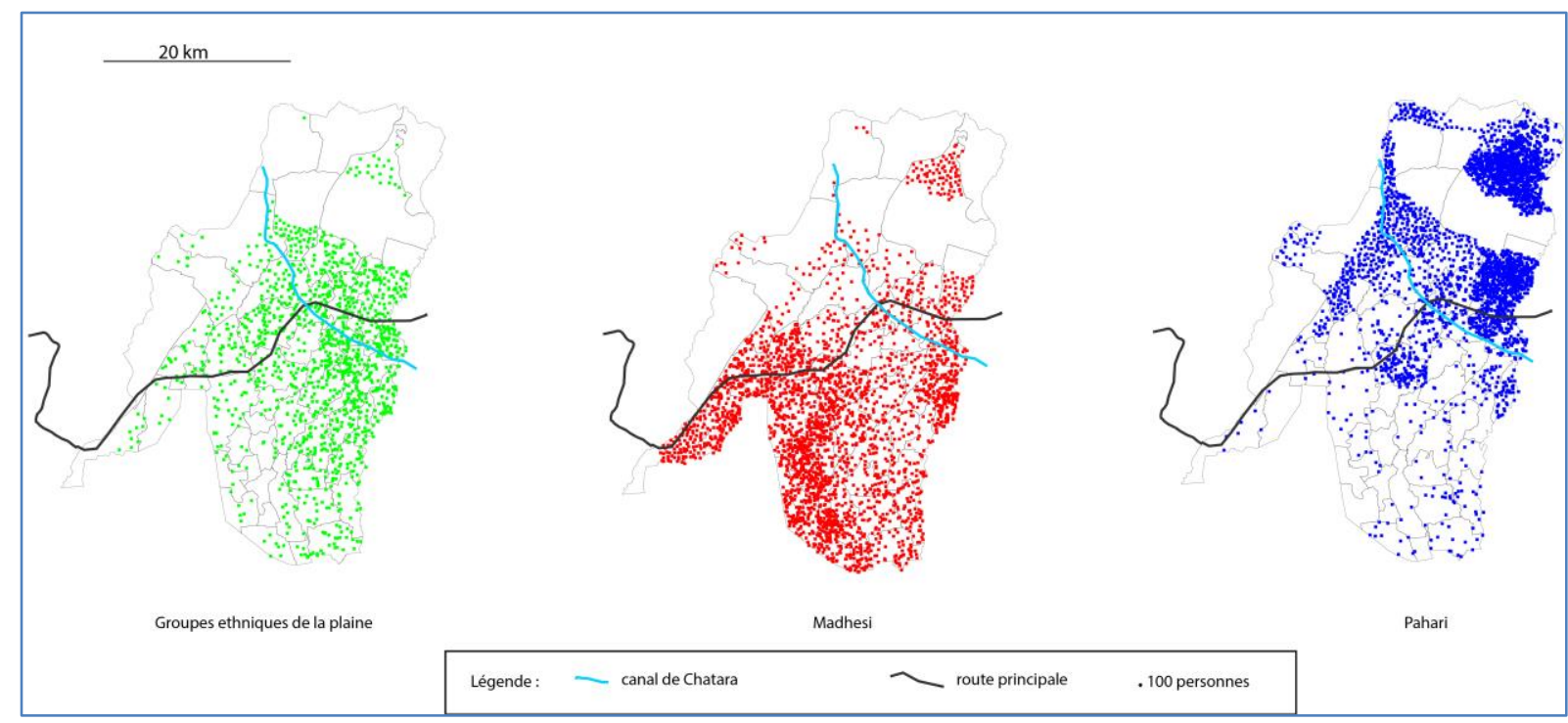

Carte 3 : Répartition spatiale de la densité des trois principaux groupes de population (ethnie de la plaine, Madhesi et Pahari) par unité administrative (VDC) dans le district de Sunsari. Réalisation : O. Aubriot (CNRS-CEH)

\subsection{Logique des aménagements}

Les logiques productives et stratégiques des projets, dites et non-dites, permettent de saisir les intérêts divergents dans le contrôle d'espaces marginaux (Faggi, Bergeron, 1990). Si le barrage et les digues de la Koshi sont gérés exclusivement par l'Inde, le SMIS et les digues sur les autres rivières sont quant à eux gérés par le Népal (selon les règles imposées par les bailleurs de fonds).

\section{a. Logique productive}

L'Inde a construit barrages et digues au Népal selon deux logiques : une logique de protection contre les crues, les rivières himalayennes dévastant fréquemment son territoire ; une logique de production, à travers les canaux issus du barrage de la Koshi (n'alimentant point le Sunsari) pour satisfaire ses besoins immenses d'irrigation.

Au Népal, l'endiguement de la Koshi permet la création d'un nouvel espace protégé (celui du 'pays de la Koshi', nom donné à l'ouest du district qui était régulièrement inondé par la rivière au lit changeant) «c'était la première fois que l'on se sentait protégés ${ }^{9}$ ». L'irrigation est également cruciale pour la production rizicole du Népal. Le réseau d'irrigation SMIS permet d'alimenter idéalement une vaste superficie grâce à l'utilisation de l'eau de la Koshi, jusque-là inexploitée. L'idéologie des Gorkhali se poursuit donc, non plus à travers des politiques foncières, mais des aménagements structuraux lourds financés par l'aide bilatérale dans un premier temps puis internationale.

b. Quelle logique stratégique des aménagements ?

Pour le premier ministre de l'union indienne nouvellement indépendante, les barrages sont "les nouveaux temples de l'Inde". La formule de Nehru érigeant l'ingénierie au même rang que les dieux, fait de l'ingénieur un acteur central et positionne en paradigme dominant la maîtrise de la nature par la technique. Le barrage de la Koshi fait en outre ressortir la suprématie politique de l'Inde, qui se matérialise par son emprise territoriale et son contrôle de l'eau au Népal : située en aval, elle est pourtant gestionnaire et principal bénéficiaire du projet localisé en territoire népalais.

\footnotetext{
${ }^{9}$ Interview d'un Madhesi de Sripur, avril 2013.
} 
Pour le Népal, les logiques sont multiples. Il s'agit d'asseoir le pouvoir royal, nouvellement restitué, et d'annexer pleinement la plaine à l'aide de technologies modernes et d'institutions étatiques. On se trouve dans la perspective de Stuart Elden (2010 cité par Giraut, 2013), qui voit dans le territoire une combinaison de contrôle économique et stratégique. La logique technocratique domine la perception de l'aménagement du territoire : les ingénieurs raisonnent en termes cartographique, topographique et de génie civil (construire des canaux rectilignes, cimentés, du nord au sud, sur les zones les plus hautes afin que l'eau puisse s'écouler de façon gravitaire dans les canaux subalternes) sans consultation ou participation des populations locales, et souvent à une vaste échelle. On peut se demander si l'un des objectifs n'est pas de déstructurer les organisations locales, qui géraient l'eau collectivement, afin de promouvoir un modèle incluant les migrants et laissant les ingénieurs avoir la mainmise sur la gestion de l'eau. Par ailleurs, les ingénieurs du DoI font appel aux entrepreneurs locaux, propriétaires des moyens techniques. Ayant le monopole du savoir et des fonds permettant le contrôle des infrastructures, ils favorisent clientélisme et corruption dans la maintenance des aménagements. Les stratégies d'enrichissement personnel sont donc également à prendre en considération.

Dans les années 1960 s'opère donc une évolution de la territorialité institutionnelle par la création d'aménagements hydrauliques et d'institutions les gérant. Les populations de la plaine, Tharu et Madhesi, doivent dorénavant composer avec deux nouveaux acteurs : d'une part les ingénieurs de l'État et d'autre part les Pahari, populations des montagnes, avec qui espace et ressources naturelles doivent être partagés.

\section{Co-construction de l'organisation hydraulique par des dynamiques sociales : reflet des recompositions territoriales}

Non seulement les acteurs gérant et/ou utilisant l'eau au sein du district se multiplient, mais avec les politiques de gestion participative, ils se diversifient : le SMIS n'est plus uniquement géré par le DoI, mais interviennent également des associations d'usagers, créées en 1994. Leur implication institutionnalisée est la porte ouverte à l'expression, dans la gestion de l'eau et des infrastructures, des conflits sociaux caractérisant le Népal contemporain. En effet, les mouvements identitaires sont dorénavant centraux sur la scène politique népalaise remodelant les débats sur la définition de la nation, les droits des citoyens et la structure de l'État (Lawoti, Hangen, 2013).

\subsection{Déconstruction du territoire hydraulique unitaire}

La forte teneur en sédiments de la Koshi remet en cause la durée de vie des aménagements et augmente considérablement les coûts de maintenance. Coincé entre les digues, le lit de la rivière se surélève avec le dépôt sédimentaire, se retrouve plus haut que la plaine environnante, fragilisant les digues. De même, la capacité des canaux se réduit avec l'ensablement, laissant moins d'eau pour l'aval. Afin de délester les canaux du SMIS du sable accumulé, les ingénieurs ont sur-creusé les rivières locales pour y déverser ce sable. D'après nos enquêtes, quelques années après la mise en place de ces actions, les populations ont observé un bouleversement des équilibres hydrologiques s'incarnant dans le grossissement du flot des rivières, avec plus au sud des inondations et une érosion plus importantes.

Sur ces rivières, la politique d'endiguement a rapidement montré ses limites. En effet, l'eau n'est pas drainée correctement à cause d'obstacles que constituent les digues et autres infrastructures tels que canaux, routes, maisons, etc. Un nouveau type d'inondation "de longue durée" émerge avec l'eau qui stagne et reste "piégée" (jusqu'à 6 mois), rendant les terres inutilisables. D'après nos observations et enquêtes, afin de laisser l'eau s'échapper, les populations victimes vont rompre digues et canaux, de préférence la nuit afin d'éviter tout 
conflit avec les communautés voisines. Les zones les plus affectées par ces terres engorgées d'eau sont localisées au sud du district et sont aussi les plus pauvres ${ }^{10}$.

En parcourant le district, on s'aperçoit que certaines digues sont mal positionnées, que la logique d'ensemble qui a dû prévaloir initialement n'existe plus. Et ce, pour plusieurs raisons. Tout d'abord, les digues ont été construites successivement par des programmes indépendants, sans coordination. Ensuite, d'après les victimes d'inondations, la localisation de ces digues serait davantage liée aux intérêts de personnes influentes localement (profitant du marché que représentent ces constructions). Enfin, ces digues sont construites à partir de modèles développés pour des rivières occidentales et sont donc inadaptées au contexte himalayen : cette inadéquation est typique des équations scientifiques de la science 'orthodoxe' se voulant universelles et représentative d'une construction sociale spécifique (Forsyth, 2003).

Quant aux canaux, une nette différence s'observe également dans le sud du district : ils sont sans eau car endommagés, voire détruits et ensablés. L'eau n'arrive pas ou très peu en aval, et son accès ne peut être assuré. Les raisons de ces dysfonctionnements dans la distribution de l'eau et la maintenance du réseau sont diverses. L'une, d'après les auteurs, est l'absence de tours d'eau favorisant l'aval afin de contrer le désavantage 'naturel' des paysans en queue de branche (dans tout système gravitaire, l'aval dépend des pratiques de l'amont pour avoir l'eau). Un projet de ce type est toutefois porté actuellement par le président de l'association des usagers de la branche SS1. Il vise à établir un calendrier des cultures qui équilibre dans le temps les besoins en eau entre nord et sud de la branche, soit entre l'aval et l'amont. Une autre raison est l'ensablement excessif des canaux, réduisant la quantité d'eau disponible. Plusieurs irrigants refusent d'ailleurs de payer la taxe sur l'eau, utilisée pourtant pour l'entretien des canaux, arguant qu'ils ne le feront que lorsqu'ils recevront effectivement l'eau. Ce cercle vicieux est alimenté par des querelles intestines au sein des associations (repérées lors d'une Assemblée Générale d'association d'irrigants) et par les relations tendues entre ingénieurs et paysans. Enfin, les réparations des canaux endommagés sont bâclées, car source de profit pour plusieurs acteurs (comme pour les digues, des arrangements semblent être réalisés entre personnalités locales influentes, ingénieurs et entrepreneurs), les réparations successives permettent davantage de bénéfices.

La réalité du système d'irrigation, avec une eau qui n'arrive que peu dans les portions sud des branches, ne ressemble donc pas à ce que la cartographie laisse croire : un réseau unitaire, avec de l'eau sur son ensemble. En outre, il ressort des entretiens réalisés auprès d'ingénieurs, que peu d'entre eux se rendent dans cette région sud et qu'ils ne gèrent donc plus le système dans sa globalité bien que leur mission soit de répondre aux besoins sur tout le district. Une carte du réseau dans son ensemble (telle la carte 2) dessine un territoire idéel, en réalité tronqué : la cartographie est bien un outil du pouvoir et une représentation du territoire à gérer (Le Bourhis, 2007 ; Elden, 2010), et l'absence de carte des zones problématiques et déficitaires en eau est peut-être une façon de gommer le problème. Les dysfonctionnements hydrauliques décrits cidessus (ensablement, drainage, canaux endommagés) ont conduit au développement de solutions localisées qui ont fait perdre la cohérence globale, menant à une inégalité nord-sud d'accès à l'eau.

\subsection{Marginalisation des Madhesi et inégalité d'accès à l'eau}

Cette inégalité nord-sud recoupe en fait un découpage social, car ce sont essentiellement des Madhesi qui ont leurs terres au sud du district et qui sont les principaux affectés par les dysfonctionnements hydrauliques. Nous ne prétendons pas que cette corrélation entre inégalité

\footnotetext{
10 Les problèmes ne s'arrêtent bien-sûr pas à la frontière. Le Bihar, adjacent, est confronté à des soucis similaires.
} 
spatiale d'accès à l'eau et distinction sociale soit volontaire. Il s'agit d'une co-construction progressive de la situation par les dynamiques sociales, sur fond de conflits ethniques et sociaux. Ces conflits sont récurrents depuis la restauration de la démocratie en 1990, période à laquelle les revendications ethniques ont fleuri ; avec la fin du conflit armé qui opposa de 1996 à 2006 le gouvernement népalais aux milices maoïstes, elles se sont profondément accrues. Pour la plaine, le soulèvement Madhesi de 2007 a marqué un tournant, obligeant les dirigeants à écouter davantage leurs revendications.

Les Madhesi dénoncent aujourd'hui la marginalisation dont ils ont été victimes depuis l'unification, ainsi que la négation historique qu'elle a induite. Ecrite depuis les montagnes, la version dominante de l'histoire présente les Madhesi comme des colons indiens sans distinction de la période d'arrivée, ni des groupes présents avant l'unification : cela minimise leur position d'utilisateurs anciens de l'espace tout en remettant en cause leurs droits territoriaux. Plusieurs groupes Madhesi ont été marginalisés de longue date, preuve en est leur absence dans le premier code civil. La chute du régime Rana n'a pas mis fin à cette marginalisation, au contraire : le faible accès à l'éducation pour les Madhesi s'est poursuivi, de même que leur faible représentation dans les instances étatiques. En outre, les termes relatifs à l'accès à la citoyenneté, révisés en 1964, ont remis en cause les droits fonciers de Madhesi (Nayak, 2010) stigmatisés "d'indiens" donc d'étrangers, révision qui a précédé stratégiquement la réforme agraire : les populations de la plaine ne pouvant répondre aux nouveaux critères ont alors perdu beaucoup de terres.

La multiplication du nombre de partis politiques Madhesi, à caractère ethnique donc, reflète la diversité de définitions possibles des Madhesi (toutes les populations de la plaine, que les castes, castes et Musulmans ${ }^{11}$, castes et certaines ethnies de la plaine). Malgré cela, un ensemble d'éléments les caractérise socialement. Une nette différence socio-économique s'observe entre les hautes et basses castes, ainsi qu'une grande dépendance des basses castes et des sans terres vis-à-vis de castes plus élevées. En effet, paupérisés à l'extrême, illettrés, souvent sans documents de citoyenneté, les plus pauvres ont besoin de relais pour les questions financières et administratives. À l'inverse, nombre des membres de plus hautes castes, propriétaires ou grands propriétaires terriens, éduqués, sont impliqués dans les partis politiques. L'organisation sociale des Madhesi permet aux représentants politiques de pouvoir compter sur une base populaire importante. Or, force est de constater que leurs programmes ne s'intéressent pas au développement local, se focalisant essentiellement sur l'arène nationale et les revendications ethniques territoriales : on peut alors se demander si les discours sur les Madhesi en difficulté ne sont pas instrumentalisés à des fins purement électorales. Les principales victimes des inégalités d'accès aux ressources et des inondations sont en effet les Madhesi pauvres, la majorité ayant perdu tout espoir de reconnaissance de la part de l'État alors que la guerre du peuple puis le soulèvement de 2007 l'avaient fait naître.

Résidant principalement dans le sud du district, les Madhesi peuvent être associés à cet espace, qui constitue un véritable territoire : il est relativement homogène socialement (que des Madhesi), spatialement (essentiellement dans le sud du district) et techniquement (terres ennoyées ou sans accès à l'eau d'irrigation car moins équipées). Il s'agit ici du territoire dans sa définition d'espace approprié économiquement, idéologiquement et politiquement (Di Méo, 1998) qui, rapporté à une revendication identitaire, permet d'évoquer un espace communautaire (Jolivet, Léna, 2000), que les intéressés délimitent souvent par la route, opposant nord et sud. Ils projettent ainsi leur image mentale du groupe, leur territorialité (Rey, 2001), la route étant le symbole de la limite géographique de leur discrimination sociale.

${ }^{11}$ Dans ce texte nous incluons sous le terme de Madhesi les populations de caste de la plaine et les Musulmans. 
Se confondent ici le territoire Madhesi et l'espace touché par les dysfonctionnements hydrauliques et par l'inégalité spatiale d'accès à l'eau. Cette situation est cristallisée dans les discours qui marquent une nette opposition entre ingénieurs/fonctionnaires (pour la majorité originaires des montagnes) et Madhesi. Ces derniers arguent que l'Etat continue à les marginaliser car il ne répare pas les canaux. Du côté des fonctionnaires, des propos racistes dictés par la peur ${ }^{12}$ véhiculent des clichés sur les populations frontalières : "ils sont dangereux, n'y allez pas, c'est très risqué, ils mentent, vous pourriez disparaître...". Lorsque l'on évoque avec des ingénieurs Pahari les terres dévastées par la rupture de digue de la Koshi en 2008 et les différences de traitement observées entre les villages victimes des inondations, leurs réponses sont révélatrices des tensions entre populations. Ils invoquent des différences culturelles et de modes de vie comme explication : " la brèche de la Koshi a sans doute été provoquée par les dieux pour les punir : contre les Musulmans car ils mangent de la vache et contre les Hindous du fait de leur orthodoxie barbare". Questionnés sur le manque d'investissement dans cette zone, l'état de délabrement voire l'absence de canaux d'irrigation, ils répondent : "Ce sont des indiens originaires du Bihâr, ils n'ont qu'à aller chercher des aides là-bas puisqu'ils y sont tous les jours ${ }^{13}$ (...) nous ne voulons pas investir dans cette zone car ils détruisent tout, preuve en est l'état des infrastructures...".

On a ici un exemple très illustratif de cette co-construction par les Madhesi et les ingénieurs d'une situation de dysfonctionnement hydraulique qui est sous-tendue par des tensions sociales : les problèmes de gestion de l'eau marquent clairement un territoire Madhesi pour les ingénieurs mais aussi pour les Madhesi eux-mêmes qui peuvent ainsi appuyer leurs revendications identitaires et dénoncer leur marginalisation. De même, en s'opposant aux ingénieurs du DoI, les Madhesi et parfois certains autres agriculteurs, expriment une opposition à l'Etat à travers la gestion de l'eau. L'irrigation permet ainsi de matérialiser des tensions (sociales et de pouvoir) et sert de support à des discours renforçant les identités communautaires. Il y a donc une représentation de la réalité et une production du discours sur les problèmes hydrauliques qui sont façonnées par les conflits sociaux. Comme l'expose Sheila Jasanoff (2004), la production de savoir (ici liée aux questions hydrauliques) est co-construite par l'ordre social.

\subsection{Mosaïque de territoires et gestion de l'eau}

À l'inverse des ingénieurs et de leur territoire tronqué, des Madhesi et de leur ancrage territorial, les deux autres types d'acteurs n'ont pas de territoire homogène. On peut se demander si cette absence permet d'expliquer que la relation territoire - gestion de l'eau n'est pas aussi manifeste.

Les Tharu ne présentent pas d'opposition forte aux ingénieurs et ne sont pas non plus dans le même positionnement revendicatif que les Madhesi. Ils sont en effet qualifiés de population originelle du Téraï et leur citoyenneté n'est pas remise en cause. Beaucoup tentent de se défaire de l'image d'ethnie 'arriérée', vivant isolément et sont plutôt enclin à participer aux projets de développement. Ils réalisent en outre les changements bénéfiques que le SMIS a apportés : plusieurs cultures par an et omniprésence du riz en mousson. Quant à leur territoire, il est dorénavant fragmenté, mité par la venue des Pahari. Le concept de territoire multisitué conviendrait ici en raison de la discontinuité des espaces appropriés, reliés par des liens sociaux, politiques et économiques (Giraut, 2013). Toutefois sa construction est différente de celles présentées dans le numéro spécial de l'Espace Géographique (2013, n4) consacré à ce concept où il résulte de politiques urbaines ségrégatives, de l'existence de corridor de transhumance, de migrations internationales, ou d'une dispersion de résidences. Ici on est dans une situation de

\footnotetext{
${ }^{12}$ Le soulèvement Madhesi de 2007 a été particulièrement virulent dans cette région

13 Les Madhesi se rendent régulièrement par-delà la frontière pour les alliances matrimoniales et les liens économiques qu'ils y entretiennent.
} 
discontinuité créée par l'arrivée massive de migrants paysans installés entre les hameaux préexistants ou à leur périphérie. Les Tharu expriment clairement le sentiment d'envahissement par les Pahari, non seulement numériquement, mais aussi dans le nouveau rapport au foncier que cela a introduit. Pensant posséder beaucoup de terres et n'en réalisant pas la valeur, ayant besoin d'argent pour payer leurs dettes, les Tharu ont vendu des terres aux Pahari. Ceux-ci sont rapidement devenus les prêteurs, à qui les Tharu s'adressaient en tant que « malik » (patron) (Gerboin, 2014, p. 19), montrant bien le renversement du rapport à la terre qui s'est alors opéré. En outre, de nombreuses anecdotes nous ont été rapportées contant les duperies des Pahari qui auraient profité de l'analphabétisme des Tharus pour récupérer des terres, notamment à l'époque de la réforme agraire de 1964 (Ibid. ; Guneratne, 2002). Le territoire actuel des Tharu correspond à un territoire qui dans le passé était continu, partagé avec les Madhesi pour la partie centrale et méridionale du district. S'il «fait» territoire (Cortès, in L'espace géographique 2013/4) aujourd'hui, c'est bien en référence à ce territoire passé, dont les hameaux exclusivement Tharu maintiennent la cohérence géographique et ethnique par 'poches' à travers des liens immatériels (matrimoniaux, fêtes rituelles etc.).

Les Pahari bénéficiant de l'eau du SMIS résident quant à eux dans les parties amont des canaux et ne souffrent généralement pas de manque d'eau. Ils peuvent marquer toutefois une opposition aux ingénieurs et protester contre les dysfonctionnements hydrauliques, étant venus dans la plaine avec l'idée de pouvoir pratiquer une agriculture productive et d'avoir une vie plus facile qu'en montagnes. Il n'est pas possible de parler de territoire pour eux : d'une part, ils ne constituent pas une entité sociale cohérente, regroupant plusieurs ethnies et castes des montagnes qui ne s'identifient pas sous une telle identité. D'autre part, il n'y a pas d'appropriation géographiquement délimitée des ressources naturelles par une seule communauté, ou même par l'ensemble des Pahari, même si dans certaines localités à forte concentration, le sentiment d'appropriation par les migrants peut exister. Enfin, les arrivées plus ou moins récentes viennent également perturber le processus de territorialisation, la construction du territoire s'inscrivant nécessairement dans le temps long (Di Méo, 1998, p.108) et ne pouvant être satisfaite ici pour l'instant.

Recompositions territoriales et dynamiques sociales sont liées. Or nous avons montré que la gestion de l'eau est intimement liée également à ces deux composantes, et que son analyse pouvait de ce fait révéler les recompositions territoriales à l'œuvre. Et s'il en est ainsi, c'est bien en raison de la co-construction de l'organisation hydraulique par les dynamiques sociales que nous avons décrites.

\section{Conclusion}

Nous avons montré dans cet article combien le rapport à l'eau et à la terre avait été modifié par divers projets de territorialité institutionnelle. Ainsi, dès l'unification du pays, les politiques foncières de l'Etat central népalais ont figé le territoire et ont créé un espace ambivalent, entre pilier économique et marge politico-sociale. L'ouverture du Népal au paradigme technocratique et aux capitaux internationaux au milieu du $20^{\mathrm{e}}$ siècle va permettre à l'Etat de réaliser son idéal ancien d'une plaine rizicole peuplée. Les aménagements hydrauliques et les migrations massives des populations montagnardes vers la plaine ont eu pour conséquence de déstructurer certaines organisations territoriales locales et de modifier les dynamiques sociales au sein du district de Sunsari. Les populations de la plaine ont été (et sont toujours) à la fois acteurs de la valorisation du Téraï et victimes de stigmatisation ethnique. Cette situation de marge dans laquelle la plaine a été mise pendant deux siècles a enfanté les conflits sociaux actuels. La marge est en effet un objet géographique paradoxal, comme l'exprime Pierre Derioz (1997) quand il revient sur l'importance du temps pour les territoires à l'écart, voyant dans "leur marginalité le résultat d'un certain retard, d'une dépendance ou d'une dévitalisation, mais aussi les prémisses d'innovations ou de reconquête". 
Les dynamiques territoriales qui résultent de ces transformations ont dessiné, à partir d'un territoire continu, partagé entre Tharu et Madhesi, plusieurs territoires plus ou moins imbriqués et fruits d'un processus de reterritorialisation associé aux acteurs : celui des ingénieurs et de leur vision tronquée de territoire unifié ; celui des Madhesi homogène, base de leur revendication identitaire ; et celui des Tharu multi-situé car discontinu par la colonisation des Pahari, ces populations des montagnes n'ayant pas encore de territoire constitué. Ces dynamiques territoriales se retrouvent dans la gestion de l'eau et des infrastructures hydrauliques, ce qui permet à certains de manifester une résistance face à l'État et d'appuyer les conflits sociaux ou les revendications ethniques sur une base matérielle. Cette emprise territoriale des dysfonctionnements hydrauliques et des revendications identitaires nous permet de conclure que la co-construction de la situation hydraulique du district de Sunsari est façonnée par les dynamiques sociales en interaction.

\section{Bibliographie}

Adhikari K. K. (1984). Nepal under Jang Bahadur 1846-1877. Vol.1. Kathmandu : Buku, $364 \mathrm{p}$.

Aubriot O. (2004). "Irrigation history in central Nepal : the interface between agriculture and technology”, Water Nepal, vol. 11, n 2, p.19-43.

Bennett L., Dahal D.R., Govindasamy P., 2008, Caste, Ethnic and Regional Identity in Nepal. Further Analysis of the 2006 Nepal Demographic and Health Survey. Calverton, Maryland, USA : Macro International Inc.

Bonerandi E., Chantal G., Yassamine T. (2001). Les territoires locaux construits par les acteurs. ENS-Géophile, ENS Fontenay/Saint-Cloud, 254 p.

Dahal D.R. (1983). «Economic development through indigenous means : A case of Indian migration in the Nepal Terai », Contributions to Nepalese Studies, vol.11, n 1, p.1-20.

Dahal D.R. (2013). «Cultural territoriality in the context of modern Nepal: some examples from the Adivasi/ Janajati and Madhesi groups ». In Smadja J (ed.) Territorial changes and territorial restructurings in the Himalayas. New Delhi: Adroit Publishers, p. 145-165.

Derioz P., (1997), «Les arrière-pays entre marginalité, retard et innovation », dans Marges, périphéries et arrière-pays, Grenoble, Actes du colloque d'Annonay, Montagnes Méditerranéennes, $\mathrm{n}^{\circ}$ 6, p. 33-36.

Di Meo G. (1998). « De l'espace aux territoires : éléments pour une archéologie des concepts fondamentaux de la géographie », L’information géographique, Vol. 62, n³, p. 99-110.

Elden S. (2010). «Thinking Territory Historically ». Geopolitics, vol. 15, no 4, p. 757-761.

Faggi P., Bergeron R. (1990). «Les développements de l'irrigation dans la diagonale aride entre logique productive et logique stratégique », Revue de géographie de Lyon, Vol. $65, n^{\circ} 1$, p. 21-26.

Forsyth T. (2003). Critical Political Ecology: The Politics of Environmental Science. London, New York : Routledge, 320 p.

Gaige F. (1975). Regionalism and national Unity in Nepal. Berkeley: University of California Press, 234 p.

Gerboin E. (2014). Agriculture et migrations internationales de travail dans l'est de la plaine du Népal: entre ruptures et adaptations. Mémoire de Master Université de Toulouse II- Le Mirail, $141 \mathrm{p}$. 
Giraut F. (2005). Fabriquer des territoires : Utopies, modèles et projets. Thèse de privatdocen, Univ. Paris I Panthéon-Sorbonne, 296 p.

Giraut F. (2013). « Territoire multisitué, complexité territoriale et postmodernité territoriale : des concepts opératoires pour rendre compte des territorialités contemporaines ? », L'espace géographique, t.42, n 4 , p. 293-305.

Guneratne A. (2002). Many tongues one people: the making of Tharu identity in Nepal. Ithaca \& London : Cornell University Press, 236 p.

Gurung H. (1998). Nepal: Social demography and expressions, Kathmandu : New Era, 211p.

Lawoti M., Hangen S. (2013). Nationalism and ethnic conflict in Nepal. New York :

Routledge, 268 p.

Jasanoff S. (2004). States of knowledge : the co-production of science and social order.

London, New York : Routledge, 317 p.

Jolivet M.J., Léna P. (2000). « Des territoires aux identités », Autrepart, n ${ }^{14, ~ p . ~ 5-16 . ~}$

Krauskopff G. (1989). Maîtres et possédés. Les rites et l'ordre social chez les Tharu (Népal). Paris : Editions du CNRS, 276 p.

Krauskopff G. (2000). « From jungle to farms: a look at Tharu history ». In Krauskopff G., Meyer P.D. (dir), The kings of Nepal and the Tharu of the Tarai. Los Angeles: Rusca press, p. $25-48$.

Le Bourhis J-P. (2007). « Du savoir cartographique au pouvoir bureaucratique. La politique des risques en France 1970-2000 ». Genèses, nº 68 , p. 75-96.

Marié M.-J. (1987). «Présence du territoire : le cas d'un grand équipement hydraulique ». Cahier / Groupe Réseaux (Les Annales de la recherche urbaine) n7, p. 61-107.

Meyer K. W. (2000). «Introduction ». In Krauskopff G., Meyer_P. D. (ed.). The kings of Nepal and the Tharu of the Tarai.Vol. 2 (a Tharu trilogy). The Panjiar Collection of 50 royal documents issued from 1726 to 1971. Los Angeles \& Kirtipur : Rusca press \& CNAS, p.1523.

Mishra D.K. (2008). Trapped! Between the devil and deep waters._Dehradun: People's science institute \& SANDRP, $208 \mathrm{p}$.

Moine A. (2006). "Le territoire comme un système complexe : un concept opératoire pour l'aménagement et la géographie ». L'espace géographique, t. 35, n², p. 115-132.

Nayak (2010). « Madhesi Movement in Nepal: Implications for India ». Fellow's seminar, IDSA, Institute for Defense Studies and Analyses, Report prepared by Pranamita Barua, Research Assistant, IDSA.

Pradhan U. (1990). Property rights and state intervention in hill irrigation systems in Nepal. Cornell University, Ph.D.

Raffestin C. (1986). « Ecogenèse territoriale et territorialité ». In Auriac F., Brunet R. Espaces, jeux et enjeux. Paris : Fayard \& Fondation Diderot, p. 175-185.

Ramirez P. (2003). «L'État népalais et la transformation des paysages d'après les documents administratifs des XVIIIe et XIXe siècles ». In Smadja J. (dir.), Histoire et devenir des paysages en Himalaya : Représentations des milieux et gestion des ressources au Népal et au Ladakh. Paris : Editions du CNRS, p.317-338. 
Regmi M.C. (1978a). Land Tenure and Taxation in Nepal. Kathmandu : Ratna Pustak Bhandar, $895 \mathrm{p}$.

Regmi MC. (1978b). Thatched huts and stucco palaces: peasants and Landlords in 19th century Nepal. New Delhi : Vikas Publ. House, 173 p.

ReY V. (2001) «Introduction ». In Bonerandi E., Chantal G., YASSAMine T. (dir.) Les territoires locaux construits par les acteurs. ENS-Géophile, ENS Fontenay/Saint-Cloud, p.11-13.

Riaux J. (2015). «Quand l'histoire orale s'invite dans la gestion de l'eau. L'espace irrigué de Kerma (Tunisie) », Espaces et Sociétés, n 160-161, p. 155-172.

Smadja J. (2013). "Introduction. Legacies and current trends: characteristics of territorymaking in the Himalayan region". In Smadja J (dir.) Territorial changes and territorial restructurings in the Himalayas. New Delhi : Adroit Publishers, p. 13- 30.

Sugden F. and Gurung G. (2012), Absentee landlordism and agrarian stagnation in Nepal: a case from the eastern Terai. Kathmandu : Nepal Institute of Development Studies (NIDS), $106 \mathrm{p}$.

Trottier J, Fernandez S (2010). « Canals Spawn Dams? Exploring the Filiation of Hydraulic Infrastructure ». Environment and History, ${ }^{\circ} 16$, p. 97-123 